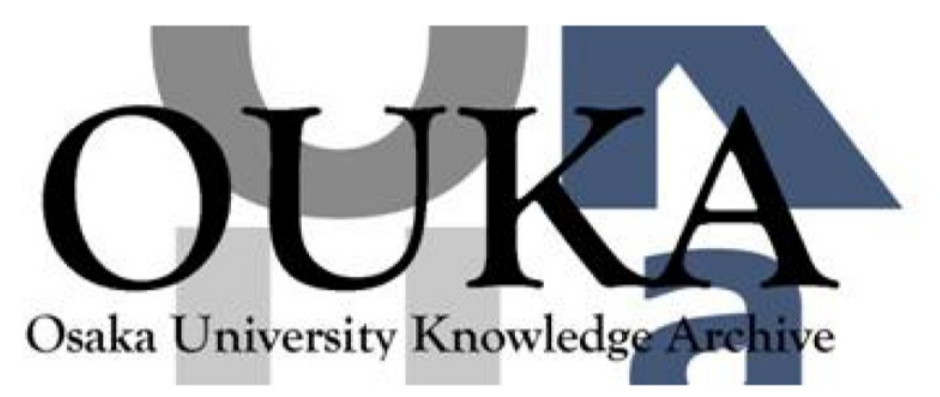

\begin{tabular}{|c|l|}
\hline Title & $\begin{array}{l}\text { Detection of glypiean-3 proteins for } \\
\text { hepatocel lular carcinoma marker using wireless- } \\
\text { electrodeless quartz-crystal microbalance }\end{array}$ \\
\hline Author(s) & $\begin{array}{l}\text { Ogi, Hirotsugu; Omori, Toshinobu; Hatanaka, } \\
\text { Kenichi et al. }\end{array}$ \\
\hline Citation & $\begin{array}{l}\text { Japanese Journal of Applied Physics. 47 p. 4021- } \\
\text { p. 4023 }\end{array}$ \\
\hline Issue Date & $2008-05-23$ \\
\hline oaire:version & AM \\
\hline URL & https://hdl. handle.net/11094/84161 \\
\hline rights & \\
\hline Note & \\
\hline
\end{tabular}

Osaka University Knowledge Archive : OUKA

https://ir. Library. osaka-u. ac. jp/

Osaka University 


\title{
Detection of Glypican-3 Proteins for Hepatocellular Carcinoma Marker Using Wireless-Electrodeless Quartz-Crystal Microbalance
}

\author{
Hirotsugu OGI, Toshinobu OMORI, Kenichi HATANAKA, Masahiko HIRAO, and \\ Masayoshi NISHIYAMA ${ }^{1}$ \\ Graduate School of Engineering Science, Osaka University, 1-3 Machikaneyama, Toyonaka, Osaka \\ 560-8531, Japan \\ ${ }^{1}$ Renovation Center of Instruments for Science Education and Technology, Osaka University \\ Machikaneyama 1-2, Toyonaka, Osaka 560-8531, Japan
}

Pure shear-wave resonances were excited and detected in 18- and 30- $\mu$ m-thick electrodeless AT-cut quartz plates in liquids using line antennas contactlessly, achieving high-frequency quartz-crystal microbalances (QCMs). Their fundamental resonance frequencies (85 and $54 \mathrm{MHz}$ ) were monitored to study interactions in real time between human glypican-3 and an anti-glypican-3 antibody: glypican-3 is a prospective protein marker for hepatocellular carcinoma. Their affinity was determined by the Langmuir kinetics. This study demonstrates the high ability of the wireless-electrodeless QCM for detection of the protein markers and development of drugs for disorders.

KEYWORDS: QCM, biosensor, glypican-3, wireless, electrodeless

\section{Introduction}

Recent advancement in proteinomics has led to identification of many marker proteins for intractable disorders, such as glypican-3 (GPC-3) protein for hepatocellular carcinoma $(\mathrm{HCC})^{1,2)}$ and the $\beta$-amyloid peptide for Alzheimer's disease. ${ }^{3)}$ Their quantitative detection allows effective treatment of such diseases. When an antigen protein is identified for a disease, it is desirable to develop a corresponding antibody medicine, which must show a high affinity with the target protein. Thus, the quantitative measurement of affinity constant between antigen-antibody molecules has been an important issue in drug development.

Conventional biosensor methods, such as the ELISA method and the surface-plasmonresonance method require detail calibrations to quantitatively determine analyte concentration and affinity values. On the other hand, a quartz crystal microbalance (QCM) ${ }^{4,5)}$ determines the affinity constant without requiring calibration in principle, because it directly detects the adsorbed mass. ${ }^{6)}$ It enables the real-time and label-free monitoring of biochemical reactions on the basis of resonance-frequency change. However, numerical simulations revealed that the sensitivity of a QCM is significantly restricted by metallic electrodes attached on the crystal. ${ }^{7,8)}$ We have then developed contactless oscillator biosensors for highly sensitive quantitative evaluation of kinetics between biomolecules. ${ }^{7-11)}$ 
Here, we intend to develop thinner (or more sensitive) electrodeless QCMs to monitor interactions between GPC-3 and an anti-GPC-3 antibody. GPC-3 is a type of glycoprotein occurring on the cell surface. A nondamaged hepatocyte does not involve GPC-3, but its level in blood increases in HCC patients. ${ }^{2)}$ Therefore, the GPC-3 protein has been recently recognized as a significant HCC marker. Generally $2 \mathrm{ng} / \mathrm{mL}$ is assumed to be the cutoff value for serum, ${ }^{2)}$ and this detection limit is achieved in the ELISA method. However, the detection limit of a conventional QCM is about $100 \mathrm{ng} / \mathrm{mL}$.

This study has two principal purposes. First, we investigate the detection limit of our QCM sensor in the detection of the GPC-3 protein. Second, we study the affinity between GPC-3 and an anti-GPC-3 antibody, which contributes to the screening process for seeking higher-affinity antibodies, and also to determine environmental conditions for enhancing affinity.

\section{Experimental Procedure}

Two AT-cut blank quartz plates were prepared. One was $30 \mu \mathrm{m}$ thick, $5 \mathrm{~mm}$ long, and $4 \mathrm{~mm}$ wide. The other was $18 \mu \mathrm{m}$ thick, $5 \mathrm{~mm}$ long, and $4 \mathrm{~mm}$ wide. Their fundamental resonance frequencies are near 54 and $85 \mathrm{MHz}$, respectively. For the gold-alkanethiol binding reaction, we deposited 9- and 5-nm-thick Au films on both surfaces of the 30 and $18 \mu \mathrm{m}$ quartz plates, respectively. The crystals were cleaned in piranha solution $\left(98 \% \mathrm{H}_{2} \mathrm{SO}_{4}: 33 \%\right.$ $\mathrm{H}_{2} \mathrm{O}_{2}=4: 1$ ), and after rinsing with ultrapure water, they were immersed in $50 \mu \mathrm{M} 5$-carboxy1-pentanethiol/ethanol solution for $3 \mathrm{~h}$. The sensor surfaces were then activated using 100 mM 1-ethyl-3-(3-dimethylaminopropyl)carbodiimide, hydrochloride (EDC) solution, and 100 mM N-hydroxysulfosuccinimide sodium salt (sulfo-NHS) solution.

The sensor crystals were then set in the handmade sensor cell ${ }^{7,11)}$ and the cell was incorporated in the homebuilt flow-injection system, as shown in Fig. 1. The micropump provided a steady flow of the carrier solution of phosphate buffer solution (PBS; pH 7.4) at a flow rate of $50 \mu \mathrm{L} / \mathrm{min}$. The switching valve selected one solution to be injected among eight vials, which flowed into the sensor cell through the degasifier. The 3-m Teflon tube column made the solution temperature stable at a set value $\left(37^{\circ} \mathrm{C}\right)$.

Tone bursts were applied to the generation antenna to generate shear-horizontal vibration of the quartz in a noncontacting manner. After the excitation, the detection antenna received reverberation signals, ${ }^{12}$ ) which entered into the superheterodyne spectrometer to measure the phase and amplitude of the received signals. ${ }^{13)}$ Frequency scanning determines resonance frequency from peak-amplitude frequency. Frequency scanning was repeated until resonance frequency became sufficiently stable (normalized fluctuation $\sim 10^{-7}$ ), and then we monitored the phase of the signal to make a quick measurement $(\sim 0.1 \mathrm{~s})$. The frequency change was determined from the linear relationship between frequency and phase near resonance frequency. ${ }^{11)}$

First, we injected the anti-GPC-3 antibody (20 or $200 \mu \mathrm{g} / \mathrm{mL}$ ) to monitor its immobiliza- 
tion behavior on the crystals surfaces, which was followed by the injection of a bovine-serumalbumin solution (BSA) $(10 \mathrm{mg} / \mathrm{mL})$ to block the remaining activated ester sites. GPC-3 protein solutions at various concentrations were then injected.

The recombinant human GPC-3 was obtained from R\&D Systems (No. 2119-GP/CF). The anti-GPC-3 antibody (host, rabbit; class, IgG) was from Santa Cruz Biotechnology Inc. (No. H-162: sc-11395). The 5-carboxy-1-pentanethiol (product number C387) and EDC (No. W001) were from Dojindo Laboratories. Sulfo-NHS (No. 56485) and BSA (No. 9048-46-8) were from Sigma-Aldrich Japan. All other chemical substances were purchased from Wako Pure Chemical Industries.

\section{Results}

Figure 2(a) shows the changes in resonance frequency during the immobilization of the anti-GPC-3 antibody and in BSA blocking. According to the Sauerbrey equation, ${ }^{6)}$ the fractional change in fundamental resonance frequency equals the ratio of the adsorbed mass of the protein to the oscillator mass. This relationship allows us to calculate the amount of the anti-GPC-3 antibody immobilized on the crystal surfaces, which is shown in Fig. 2(b).

Figure 3(a) shows the frequency responses obtained using the 30- and 18- $\mu \mathrm{m}$-thick QCMs for the injection of $5-\mu \mathrm{g} / \mathrm{mL}$ GPC-3 solution. The thinner QCM obviously enhances the frequency decrement. Figure 3(b) shows the comparison of reaction curves for various concentrations of GPC-3 solutions measured using the 18- $\mu$ m-thick QCM. Both the amount of frequency change and frequency-change rate decrease as the concentration of the analyte solution decreases.

\section{Discussion}

First, we quantitatively discuss the immobilization process. In Fig. 2(b), we observe the change in the slope at $0.25 \mathrm{pmol}$, indicating that the peptide binding of anti-GPC-3 molecules is inhibited beyond this value. This can be explained by the steric hindrance of the anti-GPC-3 antibody. The class of the anti-GPC-3 antibody used in this study was IgG, and the area of an IgG molecule is roughly estimated to be $\left.15 \times 15 \mathrm{~nm}^{2} .{ }^{14}\right)$ This value predicts the possible maximum number of molecules immobilized on the crystal surfaces because the surface area and the molecular mass (150 kDa) are known. The predicted value was about $0.3 \mathrm{pmol}$, which is close to the bending point. Therefore, nearly the whole area of the crystal was covered for 23 min and the number of binding sites decreased markedly, leading to the decrease in binding rate.

Second, we discuss the sensitivity of our electrodeless QCMs. Modification of the Sauerbrey equation gives the amount of frequency decrease for the fundamental mode $\Delta f$ of the form

$$
\Delta f=\frac{\rho_{s} v_{q}}{2 \rho_{q}} \cdot \frac{1}{d_{q}^{2}}
$$


Here, $v_{q}, \rho_{q}$, and $d_{q}$ denote the shear-wave velocity, the mass density, and the thickness of the quartz crystal, respectively. $\rho_{s}$ denotes the area density of the attached protein. $\Delta f$ indicates the sensitivity of the QCM, and it is inversely proportional to the square of the thickness of the oscillator. In conventional QCMs, 0.18-mm-thick quartz plates (9 MHz) are used. Thus, our 18- $\mu \mathrm{m}$-thick QCM allows an increase in sensitivity by 2 orders of magnitude. Indeed, the maximum frequency decrement observed for conventional QCMs is typically $200 \mathrm{~Hz}$ for the adsorption of IgG molecules, ${ }^{4}$ ) which is shown by the broken line in Fig. 3(a), demonstrating a much higher sensitivity of QCMs developed here.

Finally, we discuss the detection limit and affinity between GPC-3 and the anti-GPC-3 antibody. As shown in Fig. 3(b), the electrodeless QCM enebled the successful monitoring of binding reaction for a low-concentration GPC-3 solution. We observed the frequency change even for a $100 \mathrm{pg} / \mathrm{mL}$ analyte, which is the detection limit of our QCM at present, although we required 1-2 $\mathrm{h}$ to achieve this sensitivity to make the system stable enough. The cutoff value of GPC-3 for HCC examination is about $2 \mathrm{ng} / \mathrm{mL}$, and the QCM can be used as a simple and label-free detection tool.

The dependence of frequency-change rate on analyte concentration indicates the binding affinity between the receptor and the ligand. ${ }^{15)}$ The Langmuir kinetic analysis yields the relationship between frequency change and thermodynamic kinetic constants, which predicts an exponential decay of frequency with time. The exponential coefficient $\alpha$ is related to the reaction constants of association $k_{a}$ and dissociation $k_{d}$ as ${ }^{5)}$

$$
\alpha=k_{a} C_{A}+k_{d}
$$

Here, $C_{A}$ denotes the concentration of the analyte. Plotting $\alpha$ versus $C_{A}$ yields a line, and its slope and intercept give the reaction constants. The equilibrium constant $K_{A}$, the characteristic value for affinity, is obtained using the equation $K_{A}=k_{a} / k_{d}$. We thus determined the affinity to be $K_{A}=7.9 \times 10^{7} \mathrm{M}^{-1}$ between GPC-3 and the anti-GPC-3 antibody. This value is comparable to that between $\operatorname{IgG}$ and staphylococcus protein $\mathrm{A},{ }^{7)}$ which is known to have a high-affinity binding.

\section{Conclusions}

Electrodeless QCMs (85 and $54 \mathrm{MHz}$ ) were developed, and they were applied to detect GPC-3 protein via the immobilized anti-GPC-3 antibody on their surfaces. The detection limit was about $100 \mathrm{pg} / \mathrm{mL}$. The equiblium constant was determined from the Langmuir plot, and $K_{A}=7.9 \times 10^{7} \mathrm{M}^{-1}$ was obtained. Because electrodeless QCMs are not affected by the inertia effect of metallic electrodes, it can easily be made thinner by dry etching to achieve much higher sensitivity. 
Jpn. J. Appl. Phys.

Regular Paper

\section{Acknowledgement}

A part of this study was supported by Life Phenomena and Measurement Analysis, PRESTO, by Japan Science and Technology Agency. 


\section{References}

1) M. Capurro, I. R. Wanless, M. Sherman, G. Deboer, W. Shi, E. Miyoshi, and J. Filmus: Gastroenterology 125 (2003) 89.

2) L. M. Wright, J. T. Kreikemeier, and C. J. Fimmel: Cancer Detect. Prev. 31 (2007) 35.

3) M. Forman, J. Trojanowski, and V. Lee: Nat. Med. 10 (2004) 1055.

4) H. Muramatsu, M. Dicks, E. Tamiya, and I. Karube: Anal. Chem. 59 (1987) 2760.

5) Y. Liu , X. Yu, R. Zhao, D. Shangguan, Z. Bo and G. Liu: Biosens. Bioelectron. 18 (2003) 1419.

6) G. Sauerbrey: Z. Phys. 155 (1959) 206.

7) H. Ogi, K. Motohisa, T. Matsumoto, K. Hatanaka, and M. Hirao: Anal. Chem. 78 (2006) 6903.

8) H. Ogi, K. Motohisa, K. Hatanaka, T. Ohmori, M. Hirao, and M. Nishiyama: Jpn. J. Appl. Phys. 46 (2007) 4693.

9) H. Ogi, K. Wada, and M. Hirao: Jpn. J. Appl. Phys. 43 (2004) 3024.

10) H. Ogi, K. Motohisa, T. Matsumoto, T. Mizugaki, and M. Hirao: Biosens. Bioelectron. 21 (2006) 2001.

11) H. Ogi, K. Motohisa, K. Hatanaka, T. Ohmori, M. Hirao, and M. Nishiyama: Biosens. Bioelectron. 22 (2007) 3238.

12) H. Ogi, H. Niho, and M. Hirao: Appl. Phys. Lett. 88 (2006) 141110.

13) M. Hirao and H. Ogi: EMATs for Science and Industry: Noncontacting Ultrasound Measurements (Springer-Kluwer, Boston, 2003), p. 86.

14) V. Sarma, E. Silverton, D. Davies, and W. Terry: J. Biol. Chem. 246 (1971) 3753.

15) M. J. Eddowes: Biosensors 3 (1987) 1. 


\section{Figure Captions}

Fig. 1 Homebuilt flow-injection system.

Fig. 2 (a) Typical frequency response during immobilization of anti-GPC-3 antibody and blocking by BSA solution measured using 30- $\mu$ m-thick QCM. (b) Evolution of adsorbed mass $\Delta m$ of anti-GPC-3 antibody immobilized on the sensor surfaces.

Fig. 3 (a) Frequency responses to injection of GPC-3 solution measured using two electrodeless QCMs. The horizontal broken line indicate possible maximum frequency change, which would occur in the case of using a conventional QCM. (b) Resonance-frequency changes caused by injections of various concentrations of GPC-3 solutions detected using 85-MHz QCM. 


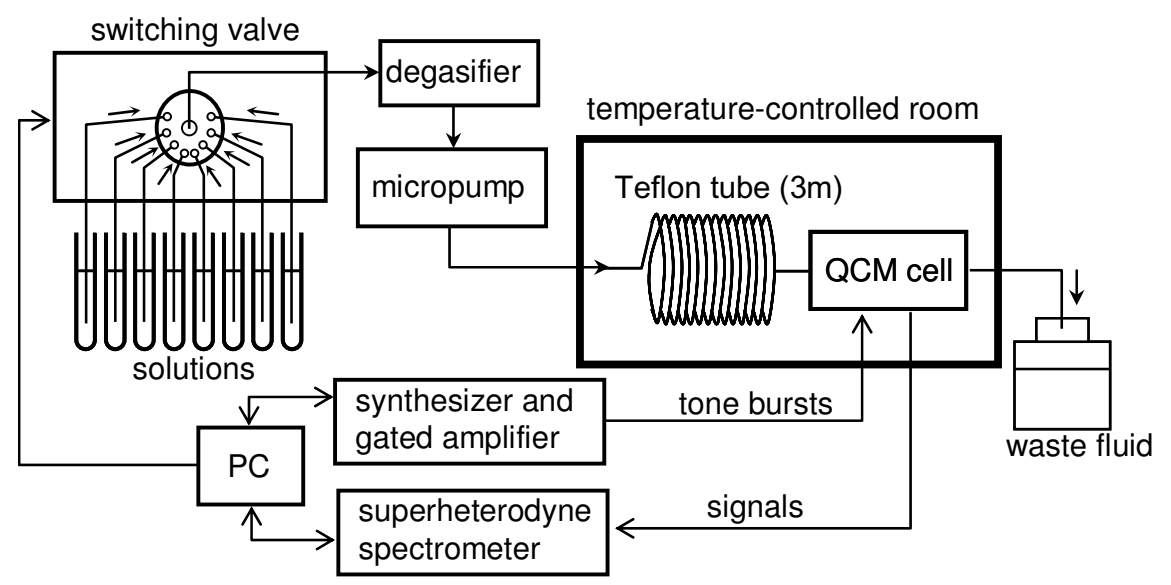

Fig. 1. 

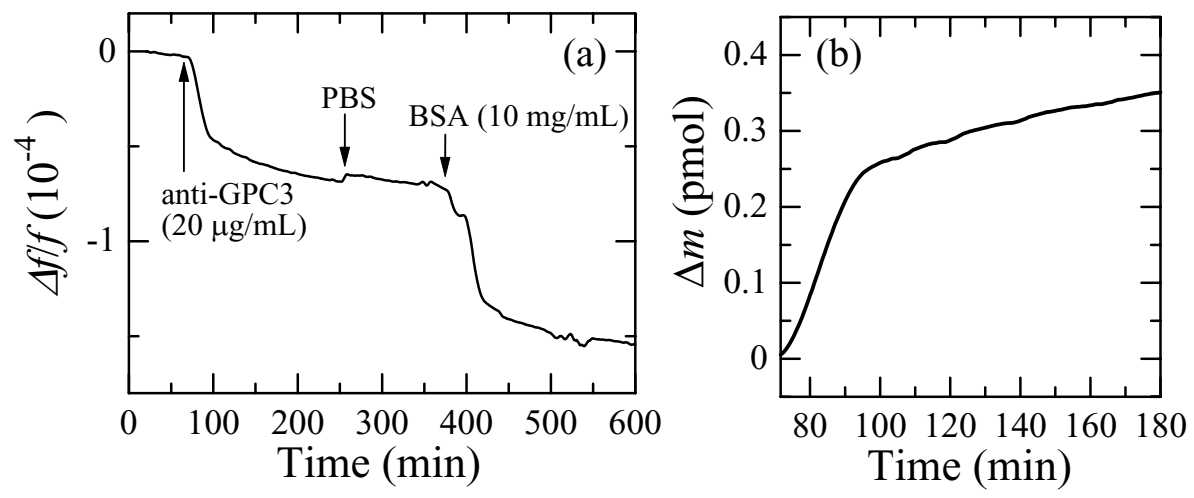

Fig. 2. 

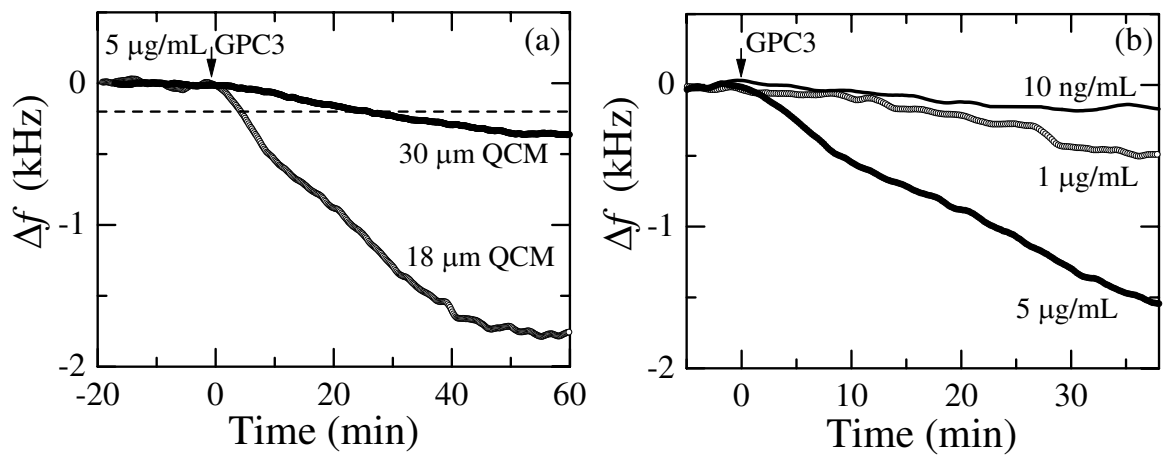

Fig. 3. 